\title{
Neuronal NLRP1 inflammasome activation of Caspase-1 coordinately regulates inflammatory interleukin-1-beta production and axonal degeneration-associated Caspase-6 activation
}

\author{
V Kaushal ${ }^{1,2}$, R Dye $^{3}$, P Pakavathkumar ${ }^{1,2}$, B Foveau ${ }^{1,2}$, J Flores $^{1,2}$, B Hyman ${ }^{4}$, B Ghett ${ }^{5}$, BH Koller ${ }^{3}$ and AC LeBlanc ${ }^{\star, 1,2}$
}

Neuronal active Caspase-6 (Casp6) is associated with Alzheimer disease (AD), cognitive impairment, and axonal degeneration. Caspase-1 (Casp1) can activate Casp6 but the expression and functionality of Casp1-activating inflammasomes has not been welldefined in human neurons. Here, we show that primary cultures of human CNS neurons expressed functional Nod-like receptor protein 1 (NLRP1), absent in melanoma 2, and ICE protease activating factor, but not the NLRP3, inflammasome receptor components. NLRP1 neutralizing antibodies in a cell-free system, and NLRP1 siRNAs in neurons hampered stress-induced Casp1 activation. NLRP1 and Casp1 siRNAs also abolished stress-induced Casp6 activation in neurons. The functionality of the NLRP1 inflammasome in serum-deprived neurons was also demonstrated by NLRP1 siRNA-mediated inhibition of speck formation of the apoptosis-associated speck-like protein containing a caspase recruitment domain conjugated to green fluorescent protein. These results indicated a novel stress-induced intraneuronal NLRP1/Casp1/Casp6 pathway. Lipopolysaccharide induced Casp1 and Casp6 activation in wild-type mice brain cortex, but not in that of $N I r p 1^{-1-}$ and Casp1 $1^{-1-}$ mice. NLRP1 immunopositive neurons were increased 25- to 30-fold in AD brains compared with non-AD brains. NLRP1 immunoreactivity in these neurons co-localized with Casp6 activity. Furthermore, the NLRP1/Casp1/Casp6 pathway increased amyloid beta peptide 42 ratio in serum-deprived neurons. Therefore, CNS human neurons express functional NLRP1 inflammasomes, which activate Casp1 and subsequently Casp6, thus revealing a fundamental mechanism linking intraneuronal inflammasome activation to Casp1-generated interleukin-1$\beta$-mediated neuroinflammation and Casp6-mediated axonal degeneration.

Cell Death and Differentiation (2015) 22, 1676-1686; doi:10.1038/cdd.2015.16; published online 6 March 2015

The lack of efficient treatment for Alzheimer disease (AD) is of high social and economical cost and a growing concern with the aging of the world's population. ${ }^{1}$ Therapies eliminating amyloid beta peptide $(A \beta)$ from $A D$ brains have unfortunately failed to stem progressive cognitive decline. These disappointing results have forced scientists to reconsider treatments against $A D$; some focusing on targeting $A \beta$ earlier in disease, while others attempting to disaggregate the Tau protein in neurofibrillary tangles (NFT). Recently, the association of several immune responsive genes with increased AD risks ${ }^{2-4}$ have additionally revived interest in a possible etiological role for inflammation in AD.

$A D$ brain inflammation is attributed to activated microglia, which remove $A \beta$, and secrete neurotoxic molecules that induce neurodegeneration. Interleukin-1-beta (IL-1 $\beta$ ), a critical component of brain neuroinflammation, is increased in $A D$ brains $^{5}$ and may contribute to $A D$ pathology by increasing amyloid precursor protein (APP) gene expression, Tau hyperphosphorylation and memory impairment. ${ }^{6}$ However, antiinflammatory therapies have not provided the expected beneficial effect in AD patients, ${ }^{7}$ suggesting that microglial inflammation may be a consequence of $A D$. Degenerating neurons are renowned initiators of brain inflammatory responses and the loss of synapses remains the best correlative marker of dementia in $A D{ }^{8}$ This has incited us to study the response of human neurons to stress and to determine whether specific neuronal molecular events were initiated that link axonal degeneration to an inflammatory response.

The active cysteinal Caspase-6 protease (Casp6), associated with axonal degeneration, ${ }^{9-13}$ is highly abundant in

${ }^{1}$ Bloomfield Center for Research in Aging, Lady Davis Institute for Medical Research, Jewish General Hospital, 3755 Ch. Cote Ste-Catherine, Montreal, QC H3T1E2, Canada; ${ }^{2}$ Department of Neurology and Neurosurgery, McGill University, 3775 University St., Montreal, QC H3A 2B4, Canada; ${ }^{3}$ Department of Genetics, 120 Mason Farm Road 5000 D, Genetic Medicine Building CB\#7264 UNC-Chapel Hill, Chapel Hill, NC 27599-7264, USA; ${ }^{4}$ Massachusetts General Hospital, Mass General Institute for Neurodegeneration, 114 16th Street, Charlestown, MA 2129, USA and ${ }^{5}$ Department of Pathology and Laboratory Medicine, Indiana University, Indianapolis, IN 46202-5120, USA

*Corresponding author: A LeBlanc, Bloomfield Center for Research in Aging, Lady Davis Institute for Medical Research, Sir Mortimer B Davis Jewish General Hospital, 3755 ch. Côte Ste-Catherine, Montréal, QC H3T 1E2, Canada. Tel: +1 514340 8260; Fax: +1 514340 8295; E-mail: andrea.leblanc@mcgill.ca

Abbreviations: $A \beta$, amyloid beta peptide; AD, Alzheimer disease; AIM2, absent in melanoma 2; ASC, apoptosis speck-like protein containing a caspase recruitment domain; BzATP, benzylated ATP; BBG, Brilliant Blue G; CA1, Cornus ammonis region 1; Casp1, Caspase-1; Casp6, Caspase-6; CD68, cluster of differentiation 68; DAMP, danger-associated molecular patterns; FDU, fluorodeoxyuridine; GFAP, glial fibrillary acidic protein; IL-1 $\beta$, Interleukin-1-beta; IPAF-1, ICE protease activating factor; MAP2, microtubule-associated protein 2; MDP, muramyl dipeptide; NLRP1, Nod-like receptor protein 1; NLRP3, Nod-like receptor protein 3; NSAID, non-steroidal anti-inflammatory drugs; PAMP, pathogen-associated molecular patterns

Received 02.7.14; revised 27.1.15; accepted 29.1.15; Edited by L Greene; published online 06.3.15 
NFT, neuropil threads, and neuritic plaques of AD brains. ${ }^{14}$ In some aged non-cognitively impaired individuals, Casp6 activity in the entorhinal cortex and $\mathrm{CA} 1$ regions of the hippocampus, ${ }^{15}$ two areas initially affected by NFT pathology in $A D,{ }^{16}$ correlates significantly with lower cognitive performance. ${ }^{17}$ The expression of active Casp6 in CA1 pyramidal neurons of mouse brains is sufficient to induce age-dependent cognitive impairment, in the absence of plaques and tangles, which suggests that active Casp6 in $\mathrm{AD}$ brains could be a major contributor to axonal degeneration and cognitive decline. ${ }^{18}$

Despite substantial evidence implicating Casp6 in AD, the pathways leading to Casp6 activation in neurons are unclear. Caspase-1 (Casp1) activates Casp6 in primary cultures of human CNS neurons. ${ }^{19}$ Inflammasome multiprotein complexes, constituted of danger sensing nucleotide-binding oligomerization domain-like receptors or the DNA sensing absent in melanoma 2 (AIM2) component, and the apoptosisassociated speck-like protein containing a caspase recruitment domain (ASC), recruit and induce Casp1 selfactivation. $^{20,21}$ Functional Nod-like receptor protein 1 (NLRP1), Nod-like receptor protein 3 (NLRP3), AIM2, and ICE protease activating factor (IPAF-1) inflammasomes have been characterized primarily in peripheral macrophages ${ }^{22}$ and CNS microglia. ${ }^{23,24}$ Recently, reports have indicated inflammasome receptor expression and activation in rodent neurons. Rat cerebellar granule neurons submitted to oxygen and glucose deprivation or reduced potassium levels increased Nirp1 mRNA levels. ${ }^{25,26}$ Nuclear NIrp1 or functional NIrp1 inflammasome complexes increased in rat cortical neurons after traumatic brain injury, stroke, and glucose-oxygen deprivation insults. ${ }^{27-31}$ Neuronal Nirp1 increased in rats submitted to spinal cord or sciatic nerve injury, ${ }^{29,32}$ and in aging rat hippocampus or ethanol treated hippocampal slice cultures. ${ }^{33,34}$ Aim2 induced pyroptosis in rat cortical neuron cultures and traumatic brain injury. ${ }^{35}$ Nirp 1 has been reported in human brain pyramidal neurons ${ }^{36}$ and inflammasome receptor mRNAs were observed in human neuron cultures and human Rasmussen's encephalitis. ${ }^{37}$

Here, we assessed which inflammasome could activate Casp 1 and subsequently Casp 6 in human primary CNS cultures. We determined which inflammasomes were expressed in naive and stressed neurons and used siRNAs and S-100 cell-free extracts treated with specific inflammasome activators, or antibody blockers, to identify the functional inflammasome. We uncovered that the NLRP1, AIM2, and IPAF-1, but not the NLRP3, inflammasomes were expressed and functional in neurons and that the NLRP1 inflammasome was responsible for Casp1 and subsequently Casp 6 activation in serum-deprived and benzylated ATP (BzATP)-stressed neurons. NLRP1 was co-localized with Casp6 activity, immunostained 25- to 30-fold more neurons in AD, and increased $A \beta_{42}$ in serum-deprived neurons. The NLRP1Casp1-Casp6 pathway was blocked in lipopolysaccharide (LPS)-treated $\mathrm{NIrp1}^{-/-}$and $\mathrm{Casp1}^{-/-}$mice brains. These results reveal a molecular cascade linking neuronal inflammasome-mediated Casp1 activation to Casp6 activation and provide unexpected novel common neuronal therapeutic targets against neuroinflammation, axonal degeneration, and cognitive impairment in AD.

\section{Results}

NLRP1, IPAF-1, and AIM2 inflammasome receptors are expressed in primary fetal human neurons. NLRP1, IPAF-1, AIM2, Casp1, and ASC inflammasome component mRNAs were detected at variable levels in naive human primary cultures of neurons, astrocytes, and microglia by RTPCR (Figure 1a). In contrast, NLRP3 was not detected in any of these cell types, despite detection in the human monocyte cell line THP-1. ${ }^{38}$ The purity of the culture was confirmed by RT-PCR with neuronal microtubule-associated protein 2 (MAP2), astrocyte glial fibrillary acidic protein (GFAP), and microglia cluster of differentiation 68 (CD68). Only neuron cultures showed a slight astrocyte contamination, as expected. Casp6 mRNA was observed in neurons, astrocytes, and THP-1 cells, but was undetectable in microglia. Quantitative assessment of inflammasome receptors with qRT-PCR showed higher levels of NLRP1 and AIM2 mRNAs in neurons than in astrocytes and microglia, while IPAF-1 levels were equivalent in all CNS cell types (Figure 1b). Consistently, NLRP1 and AIM2 proteins were higher in neurons than in astrocytes, whereas IPAF-1 protein was
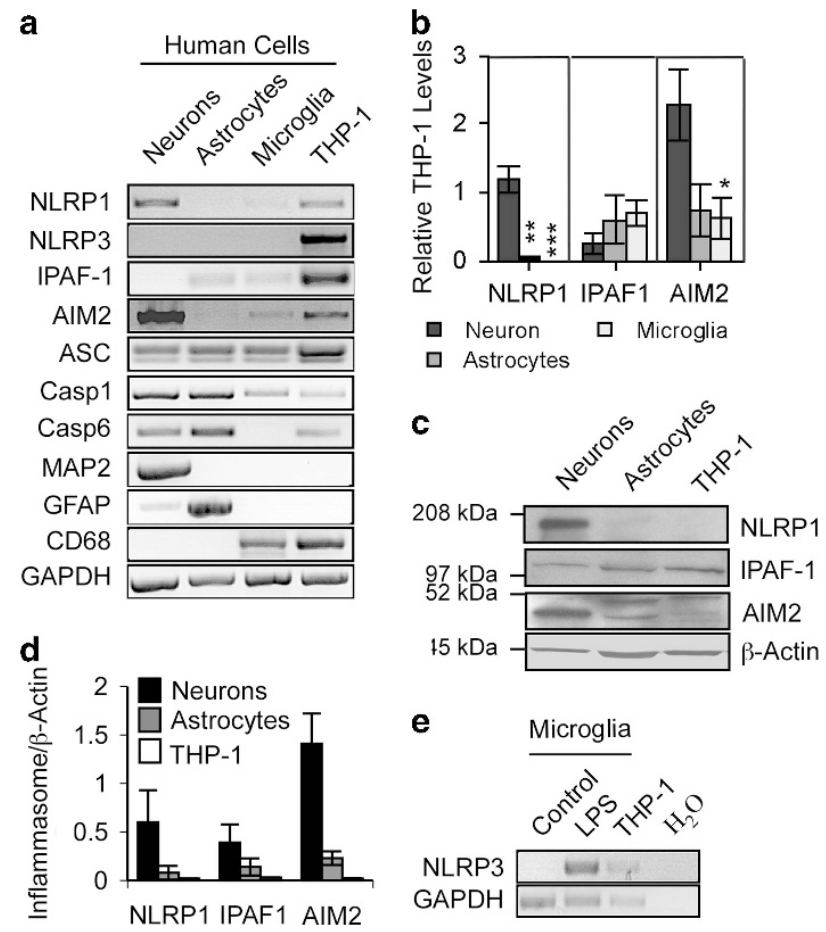

Figure 1 Expression of inflammasomes in human primary neurons. (a) Ethidium bromide agarose-stained gel of NLRP1, NLRP3, IPAF-1 and AIM2 inflammasome receptors, ASC, Casp1, Casp6, MAP2, GFAP, CD68, and GAPDH RT-PCR amplicons from human primary neurons, astrocytes, and microglia and the monocyte cell line, THP-1 as a positive control for all inflammasomes. (b) qRT-PCR of inflammasome amplicon levels corrected for GAPDH levels and expressed relative to THP-1 levels. Data represent the mean and S.E.M. of a minimum of three independent experiments. One-way ANOVA ( $P=0.0006$ for NLRP1 and $P=0.0271$ for AIM2) followed by Tukey-Kramer post hoc analysis, ${ }^{\star} P<0.05,{ }^{\star \star} P<0.01,{ }^{\star \star \star} P<0.001$. (c) Western blot of inflammasome receptors in neurons, astrocytes, and THP-1 cellular protein extracts. (d) Densitometric analysis of the western blot results of $\mathbf{c}$. Data represents the mean and S.E.M. of three independent experiments. (e) Ethidium bromide agarose gel of NLRP3 RT-PCR amplicon from untreated (control) or LPS-treated microglia 
equivalent in these cells (Figures 1c and d). Although NLRP3 was not detected by RT-PCR in any of the cell types, treatment of microglia with $100 \mathrm{ng} / \mathrm{ml}$ LPS for $24 \mathrm{~h}$, induced its expression as expected (Figure 1e). Human neurons, astrocytes, and microglia differentially express inflammasome components indicating that CNS cells could have a differential response to danger- or pathogen-associated molecular patterns (DAMPs and PAMPs).

Human neurons express functional inflammasomes. Serum deprivation of human neuron cultures, a condition previously observed to increase Casp 1 activity, ${ }^{19}$ increased NLRP1 and AIM2 mRNA levels but did not change IPAF-1 mRNA levels, whereas NLRP3 mRNA levels remained undetectable (Figures 2a and b). To assess inflammasomes functionality in neurons, a cell-free system consisting of isolated cytosol (S-100) from serum-deprived neurons, as previously described for THP-1 cells, ${ }^{38}$ was treated with NLRP1 agonist, muramyl dipeptide (MDP), AIM2 agonist polydAT, or IPAF-1 agonist recombinant flagellin and incubated at $30^{\circ} \mathrm{C}$ for $1.5 \mathrm{~h}$, and tested for Casp1 activation (Figure 2c). The incubated S-100 extracts untreated with
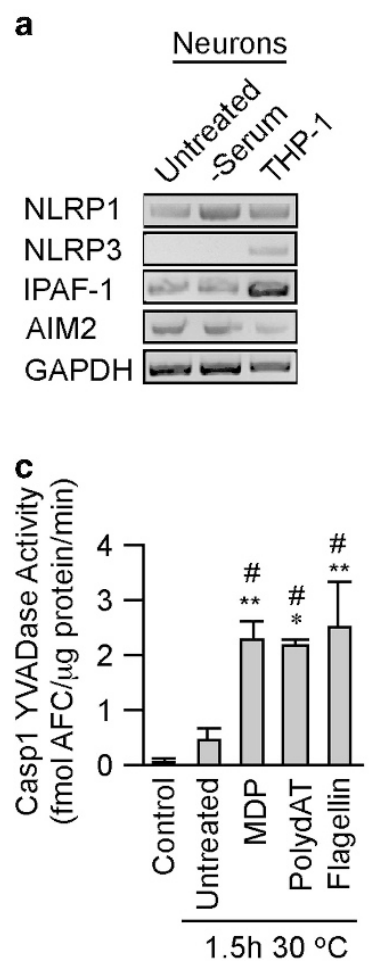

Figure 2 Functional inflammasomes in serum-deprived human neurons. (a) Ethidium bromide agarose gels of the NLRP1, NLRP3, IPAF-1, AIM2, and GAPDH RT-PCR amplicons from untreated and serum-deprived neurons compared with THP-1 cells. (b) NLRP1, IPAF-1, and AIM2 mRNA levels quantified relative to GAPDH mRNA levels by qRT-PCR in untreated and serum-deprived neurons. Statistical significance was assessed with a paired $t$-test. ${ }^{*} P<0.05,{ }^{* *} P<0.01$. (c and d) Casp1 YVADase activity (c) and IL-1 $\beta$ (d) in S-100 cytosolic fractions isolated from serum-deprived primary human neurons (control) incubated without (untreated at $1.5 \mathrm{~h} 30^{\circ} \mathrm{C}$ ) or with MDP, polydAT, and flagellin for $1.5 \mathrm{~h}$ at $30^{\circ} \mathrm{C}$. (c and d) One-way ANOVA followed by Tukey-Kramer post hoc analysis $\left({ }^{*} P<0.05\right.$, $\left.{ }^{* *} P<0.01\right)$ compares control with incubated samples, ${ }^{\#} P<0.05$ and ${ }^{\# \#} P<0.01$ compares untreated with treated samples. Data represent mean and S.E.M. from three to six independent experiments $(\mathbf{b}-\mathbf{d})$ inflammasome activators showed a 10-fold increase in Casp1 activity after incubation, consistent with inflammasome formation under these conditions. Likewise, non-serumdeprived neuron S-100 incubated at $30^{\circ} \mathrm{C}$ produced detectable Casp1 YVADase activity (Supplementary Figure S1a). Targeting the inflammasomes with $50 \mathrm{ng} / \mathrm{ml}$ MDP, $10 \mu \mathrm{g} / \mathrm{ml}$ polydAT, or $10 \mathrm{ng} / \mathrm{ml}$ flagellin increased Casp1 YVADase activity five-fold relative to the incubated control S-100 extracts (Figure 2c). Casp1 activation was confirmed by increased IL-1 $\beta$ in the incubated S-100 extracts (Figure 2d). Therefore, functional inflammasomes are not limited to immune cells and glia, but are also present in neurons.

The NLRP1 inflammasome regulates neuronal Casp1 activation. Incubation of serum-deprived neuronal S-100 at $30{ }^{\circ} \mathrm{C}$ significantly increased Casp 1 YVADase activity. Incubation of S-100 with anti-NLRP1 inflammasome receptor antibodies robustly reduced Casp1 YVADase activity (Figure 3a), while anti-AIM2 and anti-IPAF-1 antibodies did not, even after doubling their concentration (Supplementary Figure S1b). To assess if another type of cellular stress can induce neuronal inflammasome formation, neurons were treated with BzATP, known to activate the $\mathrm{P} 2 \mathrm{X} 7$ receptor (P2X7R) and the NLRP1 inflammasome in rat neurons. ${ }^{31}$ Human neurons and microglia, but not astrocytes, expressed P2X7R mRNA (Supplementary Figure S2a). The P2X7R was functional in neurons as evidenced by YO-PRO-1 dye uptake into neurons treated with BzATP, and inhibition of the YOPRO-1 dye uptake by P2X7R antagonist, Brilliant Blue G (BBG; Supplementary Figures S2b and S2c). Treatment of human neurons with BzATP induced a four-fold increase in NLRP1 mRNA levels, while IPAF-1 and AIM2 mRNA levels did not change (Figures $3 b$ and $c$ ). Furthermore, neurons treated with siRNA against NLRP1, which strongly reduced NLRP1 mRNA, eliminated serum deprivation-induced YVADase activity (Figure $3 d$ ) and $\mathrm{IL}-1 \beta$ (Figure $3 e$ ), validating NLRP1-mediated Casp1 activation. Similar results were achieved with BzATP-treated neurons but did not reach statistical significance. As serum-deprived (Supplementary Figure S3a) or BzATP-treated (Supplementary Figure S3b) pure astrocyte cultures did not activate Casp1, the NLRP1 increase must be neuronal. The functional activation of an NLRP1 inflammasome in serum-deprived neurons was confirmed with the ASC conjugated to green fluorescent protein (ASC-GFP). Normally present in the cytosol, ASCGFP is detected as condensed specks in cells when it associates with inflammasomes. As expected, ASC-GFP was distributed in the cytosol of normal neurons, but condensed into a speck in serum-deprived neurons (Figure 3f). However, siRNAs to NLRP1 prevented speck formation of ASC-GFP in serum-deprived conditions (Figure $3 \mathrm{~g}$ ), whereas neither siRNAs against Casp1 or Casp6 prevented speck formation (Figure 3g). Together, these results indicate that the NLRP1 inflammasome regulates Casp1 activation in serum-deprived and BzATP-treated human neurons.

NLRP1-induced Casp1 activation is responsible for Casp6 activation in human neurons: the NLRP1Casp1-Casp6 pathway. Casp1 YVADase activity occurred within 15-30 min followed closely by Casp6 VEIDase activity 
a

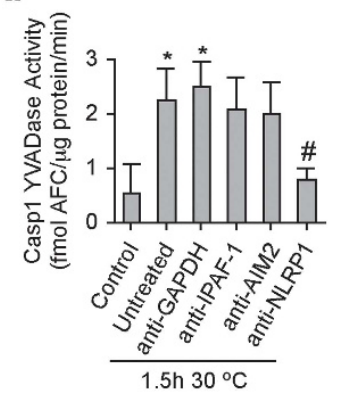

b

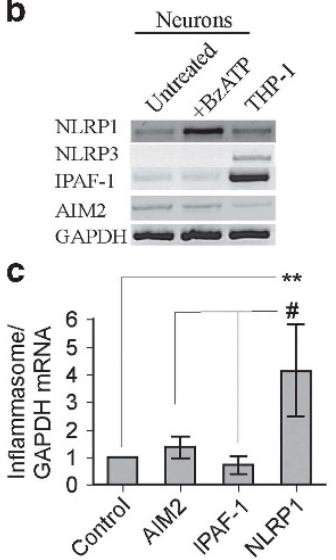

d

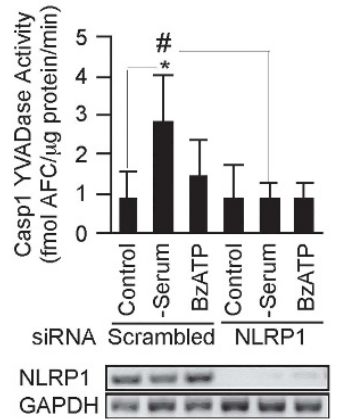

e

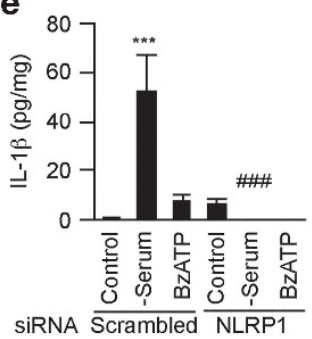

f
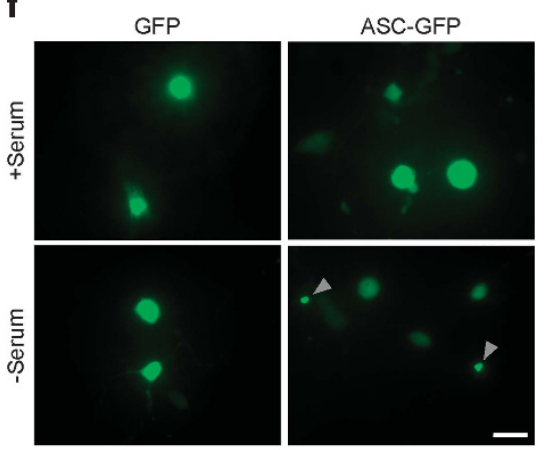

g

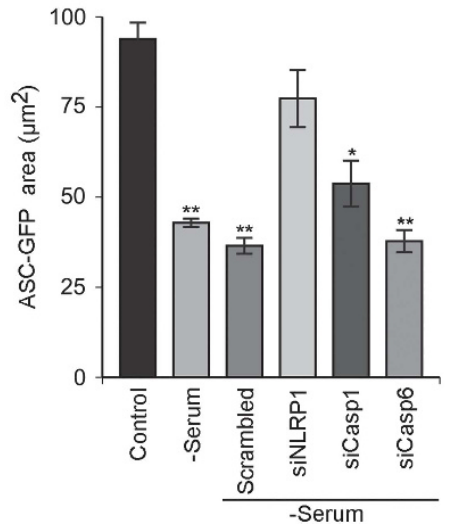

Figure 3 The NLRP1 inflammasome activates Casp1 in serum-deprived and BzATP-treated human neurons. (a) Casp1 YVADase activity in S-100 extracts from neurons exposed to serum deprivation and treated with $1 \mu \mathrm{g} / \mathrm{ml}$ antibodies against IPAF-1, AIM2, NLRP1, or GAPDH. (b and $\mathbf{c}$ ) Ethidium bromide agarose gel of RT-PCR amplicons (b) and qRT-PCR measurements (c) of NLRP1, NLRP3, IPAF-1, and AIM2 from untreated and $500 \mu \mathrm{M}$ BzATP-treated neurons. Data represent the mean and S.E.M. of four independent neuron cell cultures. One-way ANOVA $(P=0.0052)$ and Tukey-Kramer post hoc analysis ( $\left.{ }^{* \star} P<0.01\right)$ compared with control, and ${ }^{\#} P<0.05$ comparing NLRP1 with AIM2 and IPAF-1. (d) Casp1 YVADase activity in neurons treated with $1 \mu \mathrm{M}$ scrambled or NLRP1 siRNA for $72 \mathrm{~h}$ followed by 30 min serum deprivation or $500 \mu \mathrm{M}$ BzATP. Lower panel shows the effectiveness of the NLRP1 siRNAs knock down. Data represent mean and S.E.M. from four independent experiments. Repeated measures of ANOVA with a Tukey-Kramer post hoc analysis ${ }^{*} P<0.05$ comparing with control and ${ }^{\#} P<0.05$ comparing pairs of scrambled versus NLRP1 siRNAs. (e) IL- $1 \beta$ levels from samples in d. ${ }^{* * \star} P<0.001$ compared with control and ${ }^{\# \# \#} P<0.001$ comparing siNLRP1 with scrambled siRNA. (f) ASC-GFP showing specks in serum-deprived primary human neurons. Bar represents $20 \mu \mathrm{m}$. (g) Quantitation of two individual preparations of human neurons. Statistics represent a multiple $t$-test compared with control. ${ }^{\star} P<0.05,{ }^{* \star} P<0.01$

in serum-deprived (Supplementary Figure S3c) and BzATPtreated (Supplementary Figure S3d) neurons, while Casp3 DEVDase activity was observed only at $18-24 \mathrm{~h}$. Caspase activities were not observed in serum-deprived or BzATPtreated astrocytes (Supplementary Figure S3a and S3b), confirming the caspase activity to be from neurons. Casp6 activation appeared Casp1-dependent as the Casp1 YVAD peptide inhibitor prevented Casp6 VEIDase activity (Figures $4 \mathrm{a}$ and $\mathrm{d}$ ), tubulin cleaved by Casp6 (Tub $\Delta$ Casp6; Figures $4 \mathrm{~b}$ and $\mathrm{e}$ ), and IL-1 $\beta$ production (Figures $4 \mathrm{c}$ and $\mathrm{f}$ ) in serum-deprived (Figures $4 a, b$ and $c$ ) and BzATP- (Figures 4d,e and f) treated neurons. NLRP1- and Casp-1-dependent Casp6 VEIDase activation were confirmed with siRNAs against NLRP1 and Casp1, which prevented Casp6 VEIDase activity in serum-deprived neurons (Figure $4 \mathrm{~g}$ ). A similar trend was observed in BzATP-treated neurons but did not reach statistical significance. Knock down of both NLRP1 and Casp1 mRNAs with their respective siRNAs was confirmed (Figure 4h). Furthermore, serum-deprivation and BzATPinduced Tub $\Delta$ Casp6 levels were attenuated with NLRP1 and Casp1 siRNAs (Figure 4i). These results establish the NLRP1-Casp1-Casp6 pathway in human CNS neurons exposed to serum deprivation.
The NLRP1-Casp1-Casp6 pathway exists in live brains. To determine whether NLRP1 and Casp1 mediate Casp6 activation in vivo, NIrp1 $1^{-1-}, \mathrm{Casp1}^{-1-}$, or wild-type (WT) mice were subjected to a single-dose intraperitoneal (IP) injection of $5 \mathrm{mg} / \mathrm{kg}$ LPS for $6 \mathrm{~h}^{39} \mathrm{IL}-1 \beta$ increased almost 10 -fold in the brains of LPS-treated WT mice, but not in $\mathrm{NIrp1}^{-1-}$ and Casp $1^{-1-}$ LPS-treated mice (Figure 5a). Casp6 VEIDase activity increased only slightly in LPS-treated WT mice brain cortex but was significantly depressed in control and LPStreated NIrp1 null mice brains (Figure 5b). However, Casp6 VEIDase activity was not decreased in Casp1 null mice submitted to LPS. The VEIDase activity in these cortical extracts was likely due to the activity of another protease as the inhibition of Casp6 activity was confirmed by immunostaining against Tub $\Delta$ Casp6 (Figures $5 \mathrm{c}$ and d). The level of Tub $\Delta$ Casp6 did not change significantly in WT versus Nlrp $1^{-/-}$or $\mathrm{Casp1}^{-1-}$ control mouse brains. However, Tub $\Delta$ Casp6 levels increased significantly in LPS-treated WT mice versus control, and were significantly decreased in LPS-treated NIrp1 $1^{-1-}$ or Casp $1^{-1-}$ mice brains, compared with the LPS-treated WT mice brains. Tub $\Delta$ Casp6 levels were also significantly lower in the LPS-treated N/rp1 $1^{-/-}$ compared with control WT untreated brains. Casp1 cannot 


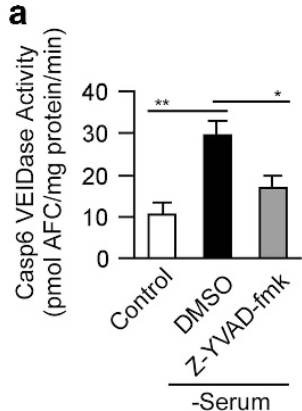

b
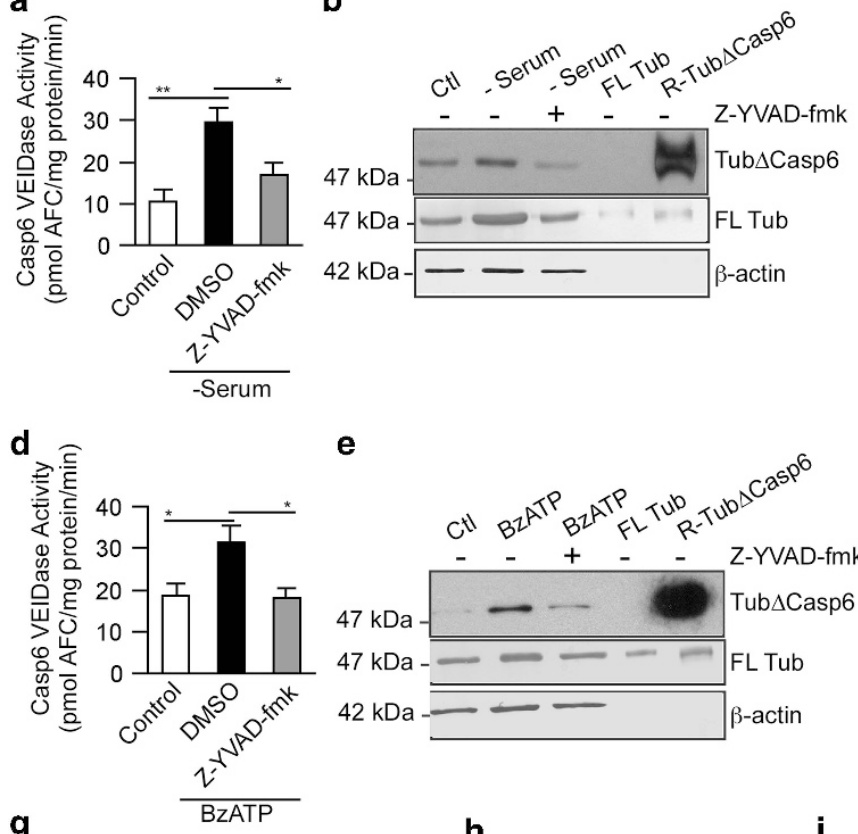

e
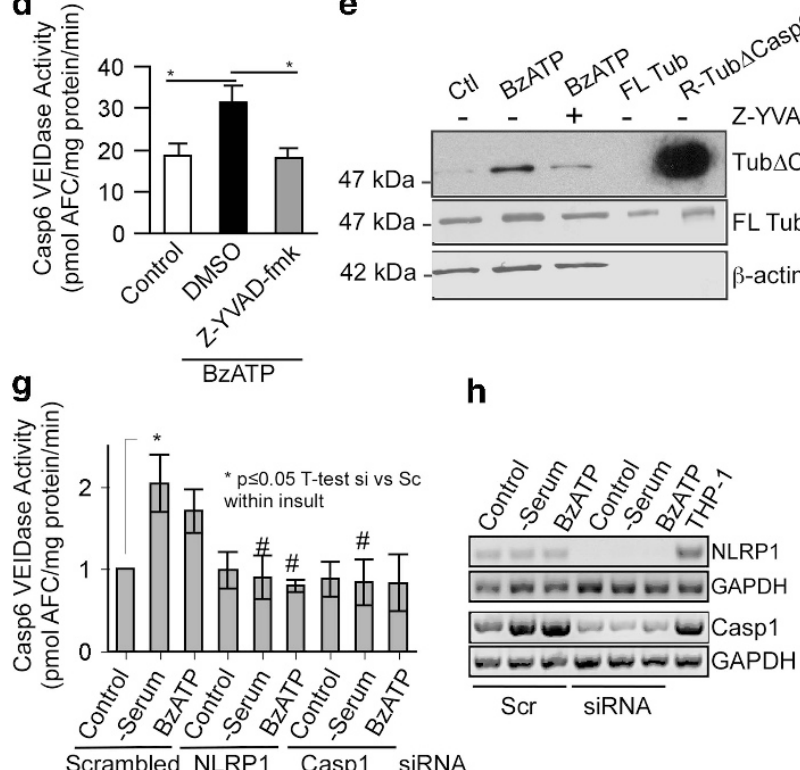

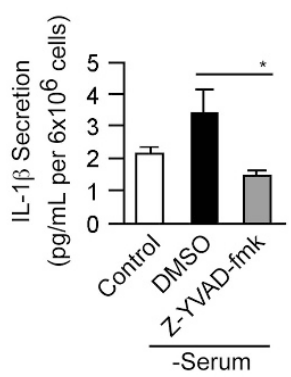

f

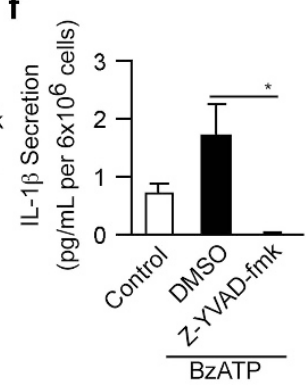

i

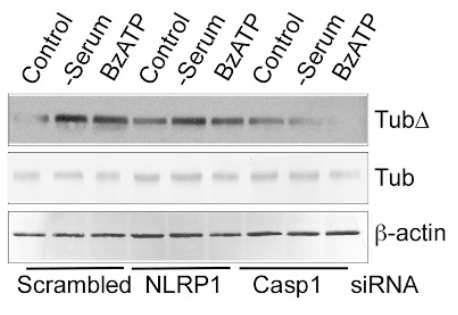

Figure 4 NLRP1 and Casp1 activate Casp6 in serum-deprived and BzATP-treated neurons. Casp6 VEIDase activity (a and d), Tub $\Delta$ Casp6, full-length tubulin (FL Tub), $\beta$-actin western blots ( $\mathbf{b}$ and $\mathbf{e}$ ) and IL- $1 \beta$ production ( $\mathbf{c}$ and $\mathbf{f}$ ) in serum-deprived ( $\mathbf{a}, \mathbf{b}$, and $\mathbf{c}$ ) or BzATP-treated (d,e, and $\mathbf{f})$ neurons in the absence (DMSO control) or presence of $5 \mu \mathrm{M}$ Casp1 Z-YVAD-fmk. Data represent the mean and S.E.M. of three $(\mathbf{a}$ and $\mathbf{c})$ and four ( $\mathbf{d}$ and $\mathbf{f})$ independent neuron cultures. One-way ANOVA $(P=0.002$ in $\mathbf{a}, P=0.004$ in c, and $P=0.02$ in $\mathbf{d}$ and f) followed by a Tukey-Kramer post hoc analysis ${ }^{*} P<0.05$ and ${ }^{* *} P<0.01$ ) relative to untreated DMSO control. (g) Casp6 VEIDase activity in neurons treated with $1 \mu \mathrm{M}$ scrambled siRNA or siRNAs against Casp1 or NLRP1 followed by normal culture conditions (control), serum deprivation or $500 \mu \mathrm{M}$ BzATP for $1 \mathrm{~h}$. Data represent the mean and S.E.M. of four independent experiments. Scrambled siRNA-treated control was arbitrarily placed at one and other values within each experiment expressed relative to the scrambled siRNA control levels. One-way ANOVA $(P=0.0106)$ followed by a post hoc Tukey-Kramer confirms increased Casp6 activity in serumdeprived neurons $\left({ }^{*} P \leq 0.05\right)$ and return to normal in siNLRP1 and siCasp1 serum-deprived neurons. Independent unpaired $t$-test shows ${ }^{\#} P<0.05$ between scrambled siRNA and siNLRP1 or siCasp1 in serum-deprived conditions, and between scrambled siRNA and siNLRP1 in BzATP-treated neurons. (h) RT-PCR of NLRP1 and CASP1 mRNA in siNLRP1 and siCASP1-treated neurons, respectively. (i) Western blot of samples in $\mathbf{g}$ with Tub $\Delta$ Casp6 and full-length tubulin antibodies

cleave Tubulin (Figure 5e) and no significant levels of Casp3 DEVDase activity were observed (Figure 5f). Together, these data confirm the presence of the NLRP1-Casp1-Casp6 pathway in vivo.

NLRP1 inflammasome receptor is increased in AD brains and neurons. NLRP1, IPAF-1, AIM2, ASC, Casp1, and Casp6 mRNAs were expressed in human brain cerebellum and cortex (Figure 6a). The increase in NLRP1 mRNA levels in human $A D$ cortex, relative to normal control brains, suggests that the NLRP1 inflammasome could lead to Casp6 activation in AD (Figure 6b). ASC, Casp1, and Casp6 mRNA levels were slightly but not significantly increased in $A D$ cortex (Figures 6c-e). As observed in human primary CNS cell cultures, NLRP3 mRNA was not detected in normal or AD brain cortex and cerebellum, despite being easily detected in THP-1 cells (Figures 6a and 4s). Immunostaining of hippocampal tissue sections revealed a robust increase of NLRP1 immunopositive cells compared with normal control subiculum (Figures $6 f$ and g). NLRP1 immunopositive staining was increased 25- to 30-fold in sporadic and familial AD subiculum (Figure $6 \mathrm{~h}$ ). NLRP1 immunopositive cells were not co-stained by GFAP, thereby excluding the presence of NLRP1 in astrocytes (Figure 6i). However, NLRP1 immunopositive cells were stained with Tau $\Delta$ Casp6, thereby confirming these cells to be neurons, some of which also contained high levels of active Casp6 (Figure 6j). Together, these data suggest that the NLRP1-Casp1-Casp6 pathway exists in human $A D$ neurons and indicate a plausible role for NLRP1 in Casp6 activation.

The NLRP1-Casp1-Casp6 pathway increases A $\beta 42$ in primary human neuron cultures. To assess whether the NLRP1-Casp1-Casp6 pathway is involved in AD 
a

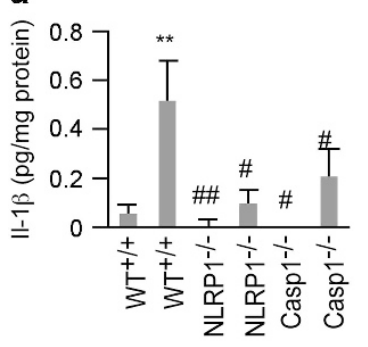

$C_{\text {LPS }}-+++++$
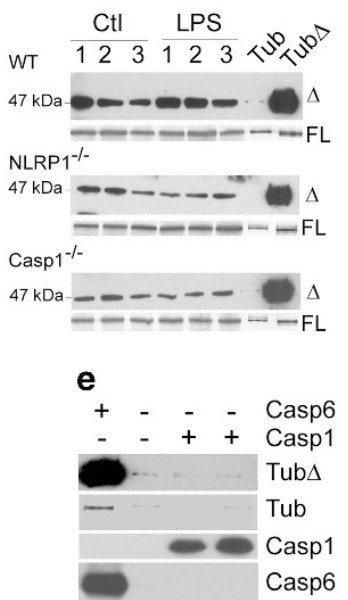

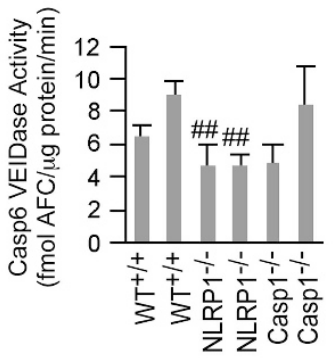

d
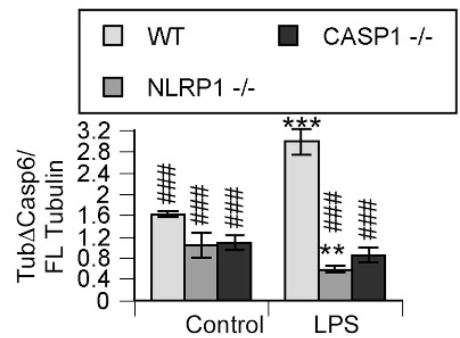

f

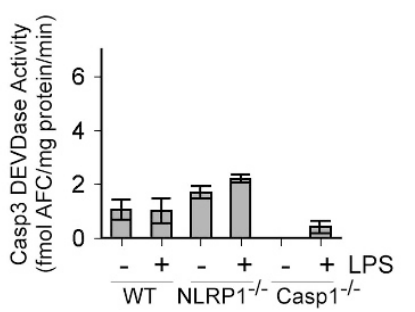

Figure 5 LPS-treated NLRP1 ${ }^{-/-}$and $\mathrm{Casp}^{-/-}$mice have reduced Casp1 and Casp6 activities. (a) Casp1-mediated IL-1 $\beta$ production, (b), Casp6 VEIDase activity (c), Tub $\Delta$ Casp6 western blots, (d) Tub $\Delta$ Casp6 relative to FL tubulin densitometry, and (f) Casp3 DEVDase activity in proteins extracted from the cortex of LPS-injected wild-type, NLRP1 null, and Casp1 null mice. Data represent the mean and S.E.M. from eight wild-type, six NLRP1 null, and three Casp1 null mice for (a,b and $\mathbf{f})$, and three different mice for ( $\mathbf{c}$ and $\mathbf{d}$ ). Statistics were performed using one-way ANOVA $(P=0.0014$ for $\mathbf{a}, P=0.002$ for $\mathbf{b}$, and $P=0.0001$ for $\mathbf{d})$ followed by a Tukey-Kramer post hoc test: ${ }^{\star *} P<0.01,{ }^{* \star *} P<0.001$ compares with WT control, ${ }^{\# \# \#} P<0.001$ compares with WT LPS (a,b and d). (e) Western blots of full-length $\alpha$-tubulin cleaved by Casp6 and Casp1

pathogenesis, primary human neurons were serum deprived after targeted knock-down expression of NLRP1, CASP1, and CASP6 with siRNAs (Figures 3 and 4). The human A $\beta$ s 38,40 , and $42\left(A \beta_{38}, A \beta_{40}, A \beta_{42}\right)$ measured in a multiplex ELISA assay, revealed a statistically significant two-fold increase in the ratio of $A \beta_{42}$ relative to total $A \beta$ peptides measured in serum-deprived neurons (Figure 7a). This was consistent with our previous experiments, ${ }^{40,41}$ and previously published data showing an increase in $A \beta_{42}$ ratio in $A D .^{42}$ Neurons pre-treated with NLRP1, CASP1, or CASP6 siRNAs lost that statistically significant increase of $A \beta_{42}$, indicating that the NLRP1-Casp1-Casp6 pathway increased the ratio of $A \beta_{42}$ peptides. NLRP1 siRNA in serum-deprived conditions did not significantly decrease $A \beta_{42}$, whereas both CASP1 and CASP6 siRNAs did, suggesting that NLRP1 may not the only inflammasome activating Casp1 in serum-deprived conditions. This was consistent with increased AIM2 mRNA levels in serum-deprived neurons (Figure 2b). Furthermore, each individual human neuron preparations were not genetically a

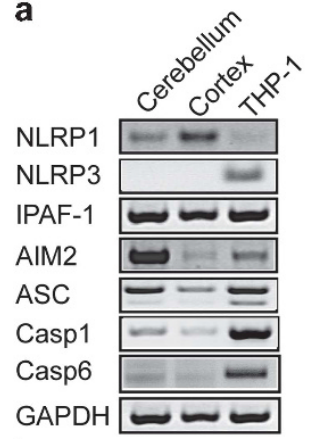

b

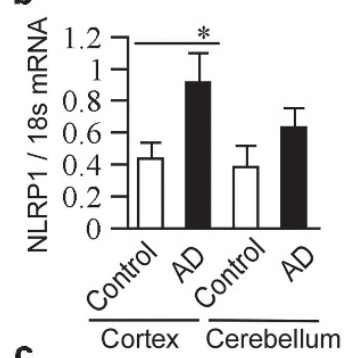

C

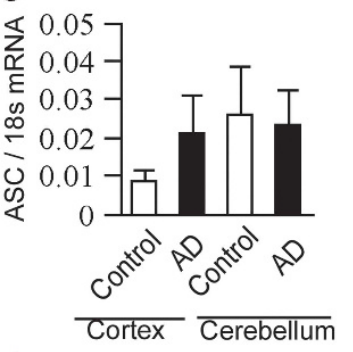

d

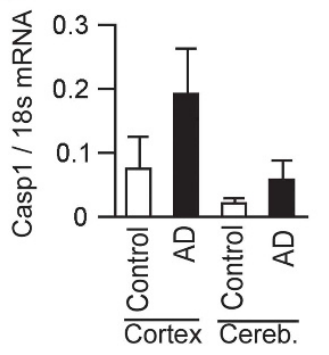

e
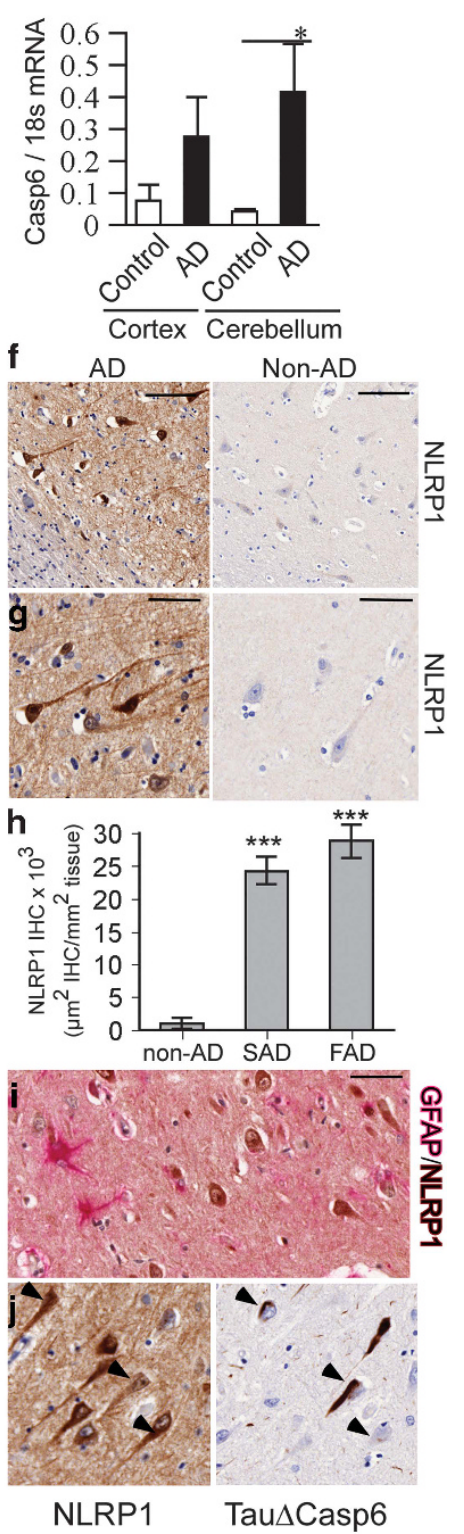

Figure 6 NLRP1 expression is increased in AD. (a) Ethidium bromide-stained agarose gel of NLRP1, NLRP3, IPAF-1, and AIM2 inflammasome receptors, ASC, CASP1, CASP6, and GAPDH RT-PCR amplicons from human adult cortex and cerebellum tissues, THP-1 cells, or water control. (b-d) qRT-PCR quantitation of cortex or cerebellar NLRP1 (b), ASC (c), CASP1 (d), and CASP6 (e) mRNAs from $A D$ and healthy control individuals. Data represent mean and S.E.M. from $12 A D$ and 4 control cases. Statistical difference was assessed by an unpaired $t$-test with Welch correction ( ${ }^{*} P<0.05,{ }^{* * *} P<0.001$ ). (f and $\mathbf{g}$ ) Low (f) and high (g) magnification micrographs of NLRP1 immunohistochemical staining in $A D$ and non-AD subiculum. Scale bar is $100 \mu \mathrm{m}$ in $\mathbf{f}$ and $50 \mu \mathrm{m}$ in $\mathbf{g}$ and $\mathbf{h}$. Density of NLRP1 immunopositive staining represented as $\mu \mathrm{m}^{2}$ positive staining per $\mathrm{mm}^{2}$ of tissue in non-AD, sporadic $A D(S A D)$, and familial $A D(F A D)$ subiculum. (i) Co-immunostaining of astrocytes with GFAP (pink) and NLRP1 (brown) in an AD brain. (j) Immunostaining of NLRP1 and Tau $\Delta$ Casp6 in $4 \mu \mathrm{m}$ consecutive sections of an AD brain. Scale is $50 \mu \mathrm{m}$ for $\mathbf{i}$ and $\mathbf{j}$

identical and could respond differentially. Nevertheless, these results provide a strong mechanistic link between the NLRP1-Casp1-Casp6 pathway and AD-associated amyloid pathology. 
a

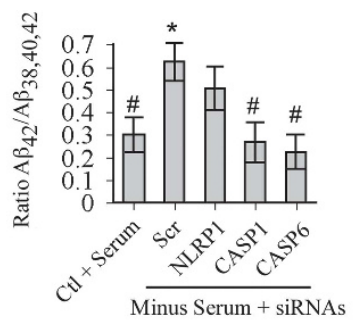

b

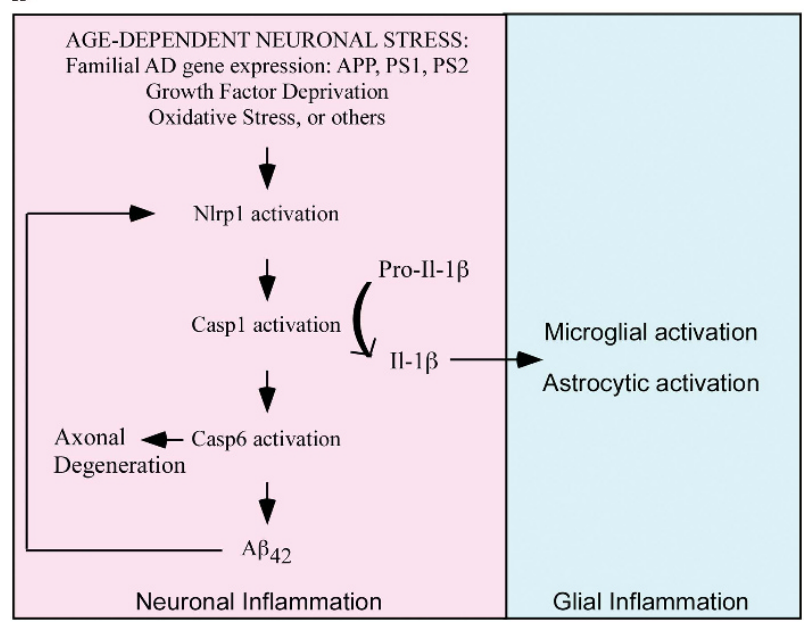

Figure 7 Nlrp1-mediated increased $A \beta 42$ ratios in serum-deprived neurons. (a) Levels of $A \beta_{42}$ relative to total $A \beta$ peptides (38, 40 , and 42 ) secreted from serumdeprived primary human neurons pre-treated with scrambled (Scr), NLRP1, CASP1, or CASP6 siRNAs. Data represents the mean and S.E.M. of four independent experiments. ANOVA $(P<0.02)$ and post hoc Dunnett analysis comparing each condition with serum-treated normal neurons $\left({ }^{*} P \leq 0.05\right)$ or with serum-deprived scrambled siRNA-treated neurons $\left({ }^{\#} P \leq 0.05\right)$. (b) Schematic diagram of a model linking intraneuronal NIrp1 inflammasome activation to Caspase-1-generated IL-1 $\beta$ mediated neuroinflammation and Caspase-6-mediated axonal degeneration and $\mathrm{A} \beta_{42}$ over-production

\section{Discussion}

In this study, we (1) show the complexity of inflammasome receptor gene expression in human brain cell types, (2) identify functional NLRP1 inflammasome in response to nonpathogenic insults of human CNS neurons, (3) identify a NLRP1-Casp1-Casp6 pathway in human neurons that links neuron-specific NLRP1 inflammasome activation with IL-1 $\beta$ mediated neuroinflammation and axonal degenerationassociated Casp6 activation, and (4) show that NLRP1 is increased in AD neurons, co-localizes with Casp6 activity, and that the NLRP1-Casp1-Casp6 pathway is involved in serum deprivation-induced $A \beta_{42}$ ratios.

Our results suggest a high degree of complexity for inflammasome-mediated responses in human brain based on the following: (1) differential gene expression of inflammasome receptors in human CNS neurons, astrocytes, and microglia primary cultures, and in normal and AD cerebellum and cortical tissues, and (2) the modulation of inflammasome receptor levels in primary cell cultures submitted to different stressors. Functional inflammasomes have been well characterized in macrophages and microglia, and recently the expression and functionality of these inflammasomes were described in rodent neurons. ${ }^{43}$ Casp1 is expressed and activated in neurons and therefore is likely to be activated via the inflammasomes. Here, we took advantage of human CNS primary neuron cultures to explore the potential impact of inflammasomes in these cell types. Human neurons expressed three (NLRP1, AIM2, and IPAF-1) of the four welldescribed immune cell inflammasome receptors and the ASC component of the inflammasome suggesting for the possibility of functional neuronal inflammasomes. These three inflammasome receptors were fully functional in a neuron cell-free system. The expression of specific inflammasome receptors varied in human primary cultures of astrocytes, microglia, and neurons. Nevertheless, neurons contained a relatively higher level of NLRP1 and AIM2 than astrocytes and microglia. Surprisingly, NLRP3 mRNA was not detected in naive human neuron, astrocyte, or microglial cultures and in $A D$ or non-AD human cortical and cerebellar tissues, NLRP3 expression was not induced in serum-deprived or BzATP-treated neuron cultures, but was induced with LPS in microglia. This latter finding is consistent with the absence of NLRP3 immunohistochemical detection in healthy human CNS tissue in contrast to its presence in peripheral tissues. ${ }^{36}$ Similar to LPS, amyloid$\beta$-peptide and mycobacteria induce NLRP3-dependent mouse microglial activation. ${ }^{23,24,44}$ Furthermore, spatial memory deficits induced in familial AD-associated mutant APP/ PS1 mice were abrogated on a null NLRP3 background. ${ }^{45}$ Although this differential expression could be species dependent, others have observed NLRP3 in human neuronal cultures, ${ }^{37}$ suggesting that culture conditions may alter the levels of inflammasome receptors in primary human neuronal cell types. Cells respond to their extracellular environment so the conditions of cell culture could strongly influence the expression of certain genes. In the study by Ramaswamy et al., ${ }^{37}$ the methods indicated that cells were passaged once for astrocytes, neurons, and microglia, whereas in our cultures, neurons were maintained as primary cells (no passage) and treated with fluorodeoxyuridine (FDU) to prevent astrocyte proliferation. Microglia were lost in FDUtreated cultures. Another difference is that Ramaswamy et al., ${ }^{37}$ cultured their cells in $10 \%$ serum whereas we used $5 \%$ decomplemented serum. It has been reported that complement can activate the NLRP3 inflammasome in dendritic cells ${ }^{46}$ and a similar mechanism in primary human neurons would explain the difference between our results. Nevertheless, as each inflammasome is activated by specific DAMPs and PAMPs, these results suggest that human neurons, astrocytes, and microglia are equipped to launch a differential response to inflammasome activating agents.

In this study, we find that the human neuronal NLRP1 inflammasome is responsible for Casp1 activation in human neuron cultures submitted to stressors. Antibodies against NLRP1 in cell-free extracts, NLRP1 siRNAs in primary neuron cultures, and NIrp1 null mice abrogate stress-mediated Casp1 activity and IL-1 $\beta$ production. Furthermore, NLRP1 is increased significantly in sporadic and familial AD hippocampal and cortical neurons suggesting an important role for NLRP1 in AD neurons, and the NLRP1-Casp1-Casp6 pathway increases the ratio of $A \beta_{42}$ relative to total $A \beta$ peptides. The recent association of genetic polymorphisms of the NLRP1 gene with AD further highlights the potential 
implication of NLRP1 in AD. ${ }^{47}$ Recently, NIrp1 has been implicated in the behavioral deficits, cell death, Casp1 activation, and IL-1 $\beta$ production of mutant presenilin $1 \Delta \mathrm{E} 9 /$ APP Swedish (PS1 $\triangle$ E9/APP ${ }^{\mathrm{Sw}}$ ) transgenic mice. ${ }^{48}$ Indeed, Nirp1 protein increased in 5- to 9-month-old PS1 $\mathrm{E} 9 / \mathrm{APP}^{\mathrm{Sw}}$ mice and injection of NIrp1 siRNAs in mice brains reduced apoptosis, Casp1, IL-1 $\beta$ production, amyloid plaques, and corrected memory deficits. Furthermore, NIrp1 siRNAs reduced the susceptibility of primary rat cortical neurons to $5 \mu \mathrm{M} \mathrm{A} \beta_{42}$. Human primary neurons are not susceptible to $\mathrm{A} \beta$ mediated toxicity, unless neurons are already vulnerable. ${ }^{9} \mathrm{We}$ therefore propose a novel model that consolidates both our results and those of Tan (Figure $7 \mathrm{~b}$ ). We hypothesize that an age-dependent neuronal stress such as a reduction in growth factors, oxidative stress, or environmental causes in sporadic $\mathrm{AD}$ or the expression of mutant APP, PS1, or presenilin 2 (PS2) in familial $A D$, induces the activation of NLRP1. Activated NLRP1 recruits and activates Casp1, which converts pro-IL- $1 \beta$ into secreted IL- $1 \beta$, thus activating a second phase of inflammation in astrocytes and microglia. ${ }^{6}$ Simultaneously, Casp1 activates Casp6-mediated axonal degeneration $^{9,11,13}$ and increased production of $A \beta_{42} \cdot{ }^{41} A \beta_{42}$ becomes toxic to already vulnerable human neurons expressing FAD-associated mutant genes or submitted to agedependent insults, or very high $A \beta_{42}$ concentrations activate the NIrp1 in rodent neurons as demonstrated by Tan. ${ }^{48}$ Note that rodents express three paralogous NIrp1 genes, whereas humans only express one NLRP1 gene. ${ }^{43}$ That the human Nirp1 protein $\mathrm{N}$-terminus contains a pyrin protein interacting domain, whereas the mouse NIrp1 contains a NR100 domain of unknown function could explain variability between rodent and human NIrp1 activation.

NLRP1 is activated by Bacillus anthracis in rodents but not in humans, but the human NLRP1 has been linked to Crohn's disease and bacterial meningitis in humans (reviewed by Walsh et al. ${ }^{43}$ ). Functional NIrp1 inflammasome has also been observed in rat traumatic brain and spinal cord injury and antiNLRP1 neutralizing antibodies reduced Casp1 activation in a mouse model of stroke. ${ }^{27-29}$ However, this is the first time that functional NLRP1 is directly observed in human neurons and in AD brains.

Another important finding of this study is the identification of neuronal NLRP1 as an upstream activator of neuroinflammation and axonal degeneration. The neuronal NLRP1-Casp1Casp6 pathway in neurons submitted to two non-pathogenic insults suggests that neuronal inflammasome activation is not necessarily activated by microbial pathogens. The NLRP1Casp1-Casp6 pathway was confirmed in vivo by the abrogation of Casp1 and Casp6 activities in LPS-treated mice brains lacking NIrp1 or Casp1. Furthermore, NLRP1 co-localized with Tau $\triangle$ Casp6 in AD neurons supporting the possibility that the activation of Casp6 occurs via NLRP1-induced Casp1 activation in AD. This NLRP1-Casp1-Casp6 pathway may explain the lack of non-steroidal anti-inflammatory drugs (NSAID) efficiency against AD because NSAID specifically target cyclooxygenase prostaglandins and do not inhibit inflammasome activity. Therefore, better therapeutic targets for AD might be NLRP1 or Casp1. Although there are three inhibitors against $\mathrm{IL}-1 \beta$ on the market, ${ }^{49}$ inhibition of IL-1 $\beta$ would help with neuroinflammation but not with
Casp6-mediated axonal degeneration. To our knowledge, there are no NLRP1 inhibitors. However, there is one Casp1 inhibitor, VX-765, in phase II clinical trials against epilepsy. ${ }^{50}$ Our results suggest that this drug might be of benefit against neuroinflammation, axonal degeneration, and cognitive impairment early in AD.

\section{Materials and Methods}

Neuronal cell cultures and cell lines. Primary human neurons were cultured as described previously ${ }^{51}$ and in accordance with a protocol approved by the McGill Institutional Review Board. Brain cortical tissues were obtained from Novagenix (Belmont, CA, USA) or the Birth Defects Research Laboratory (BRDL, University of Washington, Seattle). Briefly, 12- to 16-week-old normal fetal brains were dissociated with trypsin, treated with deoxyribonuclease I, and filtered through 130 and 70 micron nylon mesh. The dissociated cells were centrifuged for $10 \mathrm{~min}$ at 1200 r.p.m. and resuspended in minimal essential medium (MEM) with Earle's salts, supplemented with $5 \%$ decomplemented BCS, $0.1 \%$ Glucose (Sigma, St Louis, MO, USA), $1 \times$ Penicillin-Streptomycin, $1 \mathrm{mM}$ sodium pyruvate, and $2 \mathrm{mM}$ L-glutamine (Invitrogen, Carlsbad, CA, USA). Cells were seeded at a density of $3 \times 10^{6} \mathrm{cell} / \mathrm{s} / \mathrm{ml}$ on poly-lysine-coated tissue culture flasks or plates. The medium was changed every 2 days. Astrocyte proliferation was limited with the treatment of the antimitotic agent, FDU (Sigma, St Louis, MO, USA; $1 \mathrm{mM}$ ) for the first 4 days. Neuronal cultures were grown for 7-10 days before conducting experiments. The THP-1 monocyte cell line, a gift from Dr. John Hiscott (Lady Davis Institute) was used as a positive control for inflammasome components. THP-1 were grown in suspension in RPMI medium supplemented with 10\% FBS (Invitrogen). Neuronal or astrocyte cultures were exposed to serum withdrawal or 2'(3')-0-(4-Benzoylbenzoyl) adenosine-5'-triphosphate tri(triethylammonium) salt (BzATP; Sigma). Casp1 inhibitor Z-YVAD-fmk was purchased from BioMol (Philadelphia, PA, USA). Experiments were conducted on a minimum of three independent cellular preparations.

Astrocyte and microglia cultures. Purified microglia and astrocyte cultures were prepared from fetal tissue as previously described. ${ }^{51}$ However, unlike neuronal culture preparation, astrocytic and microglial media was changed after 1 day of seeding and cultures were not treated with $1 \mathrm{mM}$ FDU. After 21 days, the flasks were shaken on an orbital shaker for $15 \mathrm{~min}$ to dislodge the microglia from the underlying bed of astrocytes. Harvested microglia were centrifuged for $10 \mathrm{~min}$ at 1200 r.p.m. and resuspended in supplemented MEM (see above). The remaining bed of astrocytes were removed with $0.25 \%$ trypsin and plated at a density of $2 \times 10^{5} \mathrm{cell} / \mathrm{s} / \mathrm{ml}$. Both microglia and astrocyte cultures were used in experiments after $48 \mathrm{~h}$.

Caspase activity assays. Neuronal or astrocyte cultures were either serumdeprived or treated with $500 \mu \mathrm{M}$ BzATP for up to $24 \mathrm{~h}$. At various time intervals, neuronal proteins were extracted in cell lysis buffer (50 mM HEPES, pH 7.4, 0.1\% CHAPS, $0.1 \mathrm{mM}$ EDTA), containing a cocktail of protease inhibitors $(38 \mu \mathrm{g} / \mathrm{ml}$ AEBSF (4-(2-Aminoethyl)benzenesulfonyl fluoride hydrochloride), $0.5 \mu \mathrm{g} / \mathrm{ml}$ Leupeptin, $0.1 \mu \mathrm{g} / \mathrm{ml}$ Tosyl-L-lysyl-chloromethane hydrochloride (TLCK), $0.1 \mu \mathrm{g} / \mathrm{ml}$ Pepstatin (Sigma)). Samples were stored at $-20^{\circ} \mathrm{C}$ until ready to assay. Caspase$1,-6$ and -3 activities were measured as previously described. ${ }^{19}$ Briefly, before assessing caspase activity, protein concentration from each sample was quantified using the Bradford colorimetric protein assay and BSA standards. Neuronal protein extract $(10-20 \mu \mathrm{g})$ was mixed with a Caspase reaction mixture, containing $20 \mathrm{mM}$ PIPES, pH 7.4, $30 \mathrm{mM} \mathrm{NaCl}, 0.1 \%$ CHAPS, $1 \mathrm{mM}$ EDTA, 10\% sucrose, and $10 \mathrm{mM}$ DTT, and either $10 \mu \mathrm{M}$ Ac-YVAD-AFC for Casp1 or Ac-VEID-AFC for Casp6 or Ac-DEVD-AFC for Casp3 activities. Activity was measured using a plate reader (Bio-Tek Synergy H4, Winooski, VT, USA) with excitation at $380 \mathrm{~nm}$ and emission $505 \mathrm{~nm}$. Measurements were read every $2 \mathrm{~min}$ for $1.5 \mathrm{~h}$ and released moles of amino fluorocoumarin (AFC) were calculated from a standard curve of AFC. Specific activity for each sample was determined and standardized to protein concentration.

Conventional RT-PCR. Total RNA was isolated with TRlzol reagent (Invitrogen), according to the manufacturer's instructions and cDNA synthesis was preformed as previously described. ${ }^{52} \mathrm{PCR}$ products from synthesized CDNA were amplified with specific primers; NLRP1 Forward 5'-TCCCCCTTGGGAGTCCTCCTGAAAATG-3' and Reverse 5'-CGAGAACAGCTGGTCTTCTCCAGGGCTTC-3'; NLRP3 Forward $5^{\prime}$-AAAAGACTCATCCGTGTGCC- $3^{\prime}$ and Reverse $5^{\prime}$-TTCCTGGCATATCACA 
GTGG-3'; AIM2, Forward 5'-GCAGTGATGAAGACCATTCGTA-3' and Reverse 5'-GCTGAGTTTGAAGCGTGTTGAT-3'; IPAF-1, Forward 5'-CTCTCATGGTG GAA GCCAGTCC-3' and Reverse 5'-GACAGAGACTTGACTATGTAATCC-3'; ASC, Forward 5'-GCTGTCCATGGACGCCTTGG-3' and Reverse 5'-CATCCGT CAGGACCTTCCCGT-3'; CASP1, Forward 5'-GAAGGCATT TGTGGGAAGAA-3' and Reverse 5'-CATCTGGCTGCTCAAATGAA-3'; CASP6, Forward 5'-CGATG TGCCAGTCATTCCTT-3' and Reverse 5'-CTCTAAGGAG GAGCCATATTTTC-3'; GFAP, Forward 5'-GTGGGCAGGTGGGAGCTTGATTCT-3' and Reverse 5'-CTGG GGCGGCCTGGTATGACA-3'; MAP2, Forward 5'-GCAGTTCTCAAAGGCTAGAC-3' and Reverse 5'-TTGATCGTGGAACTCCATCT-3'; CD68, Forward 5'-GGGACCC TCAACTGCCACTCA-3' and Reverse 5'-AGAGAAGCAGGTGGGGATGGG-3'; P2X7R, Forward 5'-GAAGGCATTTGT GGGAAGAA-3' and Reverse $5^{\prime}$-CATCT GGCTGCTCAAATGAA-3'; GAPDH Forward 5'-ACCACAGTCCATGCC-3' and Reverse 5'-TCCACCACCCTGTTG-3'. Optimal annealing temperature was determined for each primer set using a temperature gradient. $2 \mu$ l of cDNA was used for each PCR reaction using Taq DNA polymerase (New England Biolabs, Whitby, ON, Canada), according to the manufacturer's protocol. The products were visualized on ethidium bromide stained $2 \%$ agarose gels.

qRT-PCR. Real-time quantitative reverse transcription (qRT)-PCR was used to measure gene transcript levels as previously described, ${ }^{53}$ using commercially available pre-validated primers (Origene, Rockville, MD, USA) for NLRP1, IPAF-1, and AIM2. THP-1 monocyte mRNA was used as internal control for experiments. Human $A D$ and control cortex and cerebellum tissues were provided by Dr. Bradley Hyman. Briefly, TRIzol reagent (Invitrogen) was used to harvest and isolate RNA according to the manufacturer's protocol. Total RNA $(1 \mu \mathrm{g})$ was reverse transcribed using 40 units of AMV-reverse transcriptase, with $1 \mathrm{mM}$ dNTPs (Promega, Madison, WI, USA) and $5 \mu \mathrm{M}$ oligo-dT (Invitrogen). qPCR was performed on CDNA using SYBR Green Taq Master Mix (Quanta Biosciences, Gaithersburg, MD, USA). mRNA amounts were calculated for each gene of interest with the housekeeping gene GAPDH or $18 \mathrm{~S}$ using the $\Delta \Delta^{\mathrm{CT}}$ method. ${ }^{54}$ The efficiency of each primer was quantified to insure no differences in amplification were due to the primer efficiencies. mRNA levels were expressed as relative fold changes.

IL-1 $\beta$ ELISA. Medium was collected from untreated neurons and neurons exposed to serum-deprivation or BzATP for $24 \mathrm{~h}$, with or without $5 \mu \mathrm{M} \mathrm{Z-YVAD-fmk}$ and DMSO. IL-1 $\beta$ levels in media were quantified using an ELISA kit, according to the manufacturer's instructions (BD Biosciences, Mississauga, ON, Canada). For mice samples, IL-1 $\beta$ levels were measured from frontal cortex tissue extracted in cell lysis buffer, using a mouse-specific ELISA kit (BD Biosciences). IL-1 $\beta$ levels were standardized to total protein concentration.

Casp1 and NLRP1 siRNA experiments. Neurons were transfected with $1 \mu \mathrm{M}$ scrambled, NLRP1 or CASP1 Accell siRNA (ThermoScientific/Fisher, Ottawa, ON, Canada) for $72 \mathrm{~h}$. Neuronal serum-deprivation or BzATP treatments were performed for $30 \mathrm{~min}$ or $60 \mathrm{~min}$ and neurons were either harvested in cell lysis buffer for protein analysis or in TRIzol (Invitrogen) to isolate RNA. Media was harvested for ELISA IL-1 $\beta$ experiments.

YO-PRO1 dye uptake. Assessment of functional P2X7R was measured by YO-PRO1 dye (Invitrogen) uptake in neurons. Neuronal cultures were seeded in a 96-well clear bottom, black-sided Costar plates (Corning, NY, USA) at a density of 50000 cells per well. Following 7 days of culturing, neurons were treated with $500 \mu \mathrm{M}$ BzATP, with or without $10 \mu \mathrm{M}$ BBG (Bio-Rad, Mississauga, ON, Canada) and $1 \mathrm{mM}$ YO-PRO1 dye. At specific times (15 min, $30 \mathrm{~min}, 45 \mathrm{~min}, 1 \mathrm{~h}, 3 \mathrm{~h}$, and $6 \mathrm{~h})$, YO-PRO1 dye fluorescence was measured using a Bio-Tek spectrofluorometer with excitation at $491 \mathrm{~nm}$ and emission $591 \mathrm{~nm}$. Background fluorescence levels from a well-containing medium only, were subtracted from all values and expressed as RFU per 50000 cells.

Cell-free inflammasome system. Neuronal inflammasome function was assessed by obtaining the S-100 cytosolic subcellular fractions as previously developed and described for THP-1 monocytes. ${ }^{38}$ Neuronal cultures were either untreated or exposed to serum-free conditions for $15 \mathrm{~min}$. Cells were washed with ice-cold PBS, lysed in ice-cold hypotonic buffer (20 mM HEPES-KOH (pH 7.5), $10 \mathrm{mM} \mathrm{KCl}, 1.5 \mathrm{mM} \mathrm{MgCl} 2,1 \mathrm{mM}$ Na-EDTA, $1 \mathrm{mM} \mathrm{Na-EGTA}, 38 \mu \mathrm{g} / \mathrm{ml}$ AEBSF, $0.5 \mu \mathrm{g} / \mathrm{ml}$ Leupeptin, $0.1 \mu \mathrm{g} / \mathrm{ml}$ Pepstatin, $0.1 \mu \mathrm{g} / \mathrm{ml} \mathrm{TLCK}$ ), and incubated on ice for $15 \mathrm{~min}$. The cellular membrane integrity was disrupted using a Dounce homogenizer (Sigma). Cell lysates were centrifuged at $1000 \times g$ to remove membrane fractions. The supernatant was harvested and centrifuged at $100000 \times g$ and the resulting S-100 supernatants were stored at $-80^{\circ} \mathrm{C}$. S-100 fractions were incubated with or without $1-2 \mu \mathrm{g} / \mathrm{ml}$ of commercially available antibodies against each inflammasome; (NLRP1 (Cell Signaling, Danvers, MA, USA), AIM2 (Novus Biologicals, Oakville, ON, Canada), and IPAF-1 (Thermo Fisher Scientific, Rockford, IL, USA)), or with specific concentrations of inflammasome activators Poly (deoxyadenylic-thymidylic) acid sodium salt (PolydAT, Sigma), MDP (Invitrogen) and recombinant flagellin (Invivogen, San Diego, CA, USA).

In vitro a-tubulin cleavage. Full length bovine $\alpha$-tubulin (Cytoskeleton Inc., Denver, CO, USA) at $600 \mathrm{ng} / \mu \mathrm{l}$ was incubated with 0.02 to $0.06 \mathrm{U} / \mu \mathrm{L}$ recombinant active Caspase-1 (BioVision, Milpitas, CA, USA) in a caspase reaction mix (20 mM DTT, $20 \mathrm{mM}$ PIPES, pH 7.4, $30 \mathrm{mM} \mathrm{NaCl}, 0.1 \%$ CHAPS, $1 \mathrm{mM}$ EDTA, and 10\% sucrose) overnight at $37^{\circ} \mathrm{C}$. Recombinant active Casp6 (200 ng/ $\mu$ l) was used as a positive control. Samples were boiled in Laemmli buffer and analyzed by western blot.

In vivo LPS mouse injections. All animal experiments were conducted in accordance with protocols approved by the McGill Animal Care Facility or Institutional Animal Care and Use Committee guidelines of the University of North Carolina at Chapel Hill. LPS from Escherichia coli 0111:B4 were purchased from Sigma. 18-22 weeks old C57BL/6 J (The Jackson Laboratory, Bar Harbor, ME, USA), NIrp1 $1^{-1-}$ (generated by Rebecca Dye in Dr. Beverly Koller's Lab, UNCChapel Hill, NC, USA), and Casp $1^{-1-}$ (The Jackson Laboratory) male mice were IP injected with a single dose of $5 \mathrm{mg} / \mathrm{kg}$ LPS for $6 \mathrm{~h}$. Mice were euthanized using $\mathrm{CO}_{2}$ and brains were extracted. Frontal cortex was dissected and was homogenized in cell lysis buffer (50 mM HEPES, pH 7.4, 0.1\% CHAPS, 0.1 mM EDTA), containing a cocktail of protease inhibitors ( $38 \mu \mathrm{g} / \mathrm{ml}$ AEBSF (4-(2-Aminoethyl)benzenesulfonyl fluoride hydrochloride), $0.5 \mu \mathrm{g} / \mathrm{ml}$ Leupeptin, $0.1 \mu \mathrm{g} / \mathrm{ml}$ TLCK, $0.1 \mu \mathrm{g} / \mathrm{ml}$ Pepstatin; Sigma) or TRIzol (Invitrogen) using a ground-glass homogenizer. Tissue was assessed for mRNA and protein analyses.

Immunohistochemistry and quantification. Non- $A D$ and $A D$ tissue was obtained with informed consent from the patients or their nearest relative and according to institutional ethical guidelines. Non-AD (1401, 4593, and 4641) and AD (1048, 1459, and 2992) brains used for immunohistochemistry were a kind gift from Dr. Catherine Bergeron (University of Toronto, Canada). Dr. Bernardino Ghetti provided familial $A D$ tissues. Tissue sections were deparaffinized, rehydrated, and treated with citrate antigen retrieval buffer $(0,01 \mathrm{M}$ Tris-Na Citrate, $\mathrm{pH} 6)$ for $20 \mathrm{~min}$ at $97^{\circ} \mathrm{C}$ in the Pascal Dako Cytomation apparatus. The immunostaining was done using the Dako Autostainer Plus automated slide processor and the EnVision Flex system (Dako, Burlington, ON, Canada) or EnVision G/2 Doublestain System (Dako) for co-staining. The antibodies used were against human NLRP1 (1/300; LifeSpan BioSciences, Inc, Seattle, WA, USA), GFAP (1/8000; Dako) and laboratory-made Tau $\Delta$ Casp6 (10635: 1/12500). Slides were counterstained with haematoxylin, dehydrated, mounted in Permount mounting medium (Fisher Scientific, Ottawa, ON, Canada) and digitally scanned with the MIRAX SCAN (Zeiss, Don Mills, ON, Canada). Using the scanned tissue sections from the Mirax digital scanner (Don Mills, ON, Canada) five images were taken at magnification $\times 20$ from three $A D$ and three non- $A D$ cases.

Familial AD cases (2002-086, 2008-073, 2006-093, 2007-093) used for quantification studies were a kind gift from Dr. Bernardino Ghetti (Indiana Alzheimer Disease Center, Indiana University Medical Center, Indianapolis, IN, USA). For NLRP1 staining quantification, ImageJ software (NIH, Bethesda, MD, USA) was used to measure the surface covered by staining in neurons. The results were expressed as NLRP1 $\mu \mathrm{m}^{2} / \mathrm{mm}^{2}$ subiculum. Statistics were performed using one-way ANOVA with a Tukey-Kramer post hoc test.

Western blotting. $25-50 \mu \mathrm{g}$ of neuronal protein or mouse cortex lysate were prepared in Laemmli buffer and electrophoresed on a $15 \%$ SDS-PAGE gel. Laboratory-made anti-Casp6p20 10630 neoepitope antibody (1:10 000) was used to detect the cleaved p20 subunit of active Casp6 and anti-tubulin cleaved by Casp6 (Tub $\Delta$ Casp6) GN60622 neoepitope antibody $(1: 10000)$ was used to detect Casp6-cleaved tubulin fragments. $\beta$-actin monoclonal antibody (Sigma, 1:5000) and full-length $\alpha$-tubulin antibody (Cell Signaling, Danvers, MA, USA, $1: 1000$ ) were used as loading controls. For the inflammasome receptors, neuronal protein were extracted in cell lysis buffer $(20 \mathrm{mM}$ HEPES, pH 7.5, $50 \mathrm{mM} \mathrm{KCl}, 150 \mathrm{mM} \mathrm{NaCl}$, $1.5 \mathrm{mM} \mathrm{MgCl} 2,1 \mathrm{mM}$ EGTA, 0.5\%, NP-40, 1 mM DTT, $0.159 \mathrm{mM}$ AEBSF, $1.458 \mathrm{mM}$ pepstatin, $1.17 \mathrm{mM}$ leupeptin, $0.27 \mathrm{mM}$ TLCK), microcentrifuged $15 \mathrm{~min}$ at high 
speed, and $50 \mu \mathrm{g}$ supernatant protein loaded on $10-15 \%$ SDS-PAGE. Western blots were probed with anti-NLRP1 (Enzo Life Sciences, Farmingdale, NY, USA; diluted $1: 1000)$, anti-AIM2 (1:1000 dilution from Cell Signaling, Beverly, MA, USA), antiIPAF-1 (anti-CLAN 1/1000 from Pierce Thermo Scientific, Rockford, IL, USA). Secondary antibodies conjugated with HRP were from Dako (anti-rabbit) and GE HealthCare (Baie-D'Urfe, QC, Canada) (anti-mouse). ImageJ software was used to quantify band intensities in triplicate gels.

Analysis of secreted $\mathbf{A} \boldsymbol{\beta}$ peptides. Human primary neurons were treated with $1 \mu \mathrm{M}$ Accell siRNAs against NLRP1, CASP1, and CASP6 (controlled with scrambled siRNAs) for $72 \mathrm{~h}$. Neurons were then serum-deprived for $24 \mathrm{~h}$. Media was collected and frozen until analysis with the Mesoscale Discovery (MSD; Rockville, MD, USA) human A $\beta$ panel 1 (6E10) kit as directed by the manufacturer.

ASC-GFP experiments. The pCl-ASC-HA (human ASC) construct, deposited by Kate Fitzgerald (UMass., MA) ${ }^{55}$ was obtained from Addgene (Cambridge, MA, USA). The PCl-ASC-HA was used as a template to amplify the human ASC with ASC_Bglll_For primer 5 '-CCGCTAAGATCTGCCATGGGGCGCGCGCGCGA CGCCATCC-3' and ASC_Agel_Rev primer 5'-ACGCGAACCGGTGACCGCTCCAG GTCCTCCACC-3'. The amplicon was cloned into the pEGFP-N1 construct to produce the pASC-EGFP expression construct. Neurons were treated with NLRP1, CASP1, or CASP6 siRNAs as described above and transfected with the ASC-GFP. After $24 \mathrm{~h}$, neurons were serum deprived and pictures of GFP-positive neurons taken under the fluorescence microscope. The area of fluorescence was quantitated. The experiment was repeated in two independent neuron preparations with 30-60 neurons per condition.

Statistical analyses. All data are presented as the mean of each treatment group \pm S.E.M. The statistical significance of differences between experimental groups was analyzed using InStat software (GraphPad Software, La Jolla, CA, USA) as indicated in the figure legends.

Acknowledgements. We thank Marc-André Déry for generating the ASC-GFP construct. The Canadian Foundation of Innovation, Canadian Institutes of Health Research (CIHR) MOP-243413-BCA-CGAG-45097, and the JGH Foundation (ALB), and NIH Grant P30 AG010133 (BG) supported this work. Dr. Vikas Kaushal was a recipient of McGill's CIHR Neuroinflammation Training Program award (2010-2012); Prateep Pakavathkumar is the recipient of a Fonds de recherche Québec-Santé scholarship (2011-2013) and the FRSQ-Alzheimer Society of Canada doctoral award (2014-2016).

1. Reitz C, Brayne C, Mayeux R. Epidemiology of Alzheimer disease. Nat Rev Neurol 2011; 7: 137-152.

2. Kamboh Ml, Demirci FY, Wang X, Minster RL, Carrasquillo MM, Pankratz VS et al. Genome-wide association study of Alzheimer's disease. Transl Psychiatry 2012; 2: e117.

3. Hollingworth P, Harold D, Sims R, Gerrish A, Lambert JC, Carrasquillo MM et al. Common variants at $A B C A 7, M S 4 A 6 A / M S 4 A 4 E, E P H A 1, C D 33$ and CD2AP are associated with Alzheimer's disease. Nat Genet 2011; 43: 429-435.

4. Zhang B, Gaiteri C, Bodea LG, Wang Z, McElwee J, Podtelezhnikov AA et al. Integrated systems approach identifies genetic nodes and networks in late-onset Alzheimer's disease. Cell 2013; 153: 707-720.

5. Griffin WS, Stanley LC, Ling C, White L, MacLeod V, Perrot LJ et al. Brain interleukin 1 and S-100 immunoreactivity are elevated in Down syndrome and Alzheimer disease. Proc Natl Acad Sci USA 1989; 86: 7611-7615.

6. Shaftel SS, Griffin WS, O'Banion MK. The role of interleukin-1 in neuroinflammation and Alzheimer disease: an evolving perspective. J Neuroinflammation 2008; 5: 7.

7. Jaturapatporn D, Isaac MG, McCleery J, Tabet N. Aspirin, steroidal and non-steroidal antiinflammatory drugs for the treatment of Alzheimer's disease. Cochrane Database Syst Rev 2012; 2: CD006378.

8. Terry R, Masliah E, Salmon D, Butters N, DeTeresa R, Hill R et al. Physical basis of cognitive alterations in Alzheimer's disease: synapse loss is the major correlate of cognitive impairment. Ann Neurol 1991; 30: 572-580.

9. Sivananthan SN, Lee AW, Goodyer CG, LeBlanc AC. Familial amyloid precursor protein mutants cause caspase-6-dependent but amyloid beta-peptide-independent neuronal degeneration in primary human neuron cultures. Cell Death Dis 2010; 1: e100.

10. Simon DJ, Weimer RM, McLaughlin T, Kallop D, Stanger K, Yang J et al. A caspase cascade regulating developmental axon degeneration. J Neurosci 2012; 32: 17540-17553.

11. Nikolaev A, McLaughlin T, O'Leary DD, Tessier-Lavigne M. APP binds DR6 to trigger axon pruning and neuron death via distinct caspases. Nature 2009; 457: 981-989.
12. Cusack CL, Swahari V, Hampton Henley W, Michael Ramsey J, Deshmukh M. Distinct pathways mediate axon degeneration during apoptosis and axon-specific pruning. Nat Commun 2013; 4: 1876

13. Uribe V, Wong BK, Graham RK, Cusack CL, Skotte NH, Pouladi MA et al. Rescue from excitotoxicity and axonal degeneration accompanied by age-dependent behavioral and neuroanatomical alterations in caspase-6-deficient mice. Hum Mol Genet 2012; 21: 1954-1967.

14. Guo H, Albrecht S, Bourdeau M, Petzke T, Bergeron C, LeBlanc AC. Active caspase- 6 and caspase-6-cleaved tau in neuropil threads, neuritic plaques, and neurofibrillary tangles of Alzheimer's disease. Am J Pathol 2004; 165: 523-531.

15. Albrecht S, Bogdanovic N, Ghetti B, Winblad B, LeBlanc AC. Caspase-6 activation in familial alzheimer disease brains carrying amyloid precursor protein or presenilin $\mathrm{i}$ or presenilin II mutations. J Neuropathol Exp Neurol 2009; 68: 1282-1293.

16. Braak H, Alafuzoff I, Arzberger T, Kretzschmar H, Del Tredici K. Staging of Alzheimer disease-associated neurofibrillary pathology using paraffin sections and immunocytochemistry. Acta Neuropathol (Berl) 2006; 112: 389-404.

17. Ramcharitar J, Afonso VM, Albrecht S, Bennett DA, LeBlanc AC. Caspase- 6 activity predicts lower episodic memory ability in aged individuals. Neurobiol Aging 2013; 34: 1815-1824.

18. LeBlanc AC, Ramcharitar J, Afonso V, Hamel E, Bennett DA, Pakavathkumar $\mathrm{P}$ et al. Caspase- 6 activity in the CA1 region of the hippocampus induces age-dependent memory impairment. Cell Death Differ 2014; 21: 696-706.

19. Guo H, Petrin D, Zhang Y, Bergeron C, Goodyer CG, LeBlanc AC. Caspase-1 activation of caspase-6 in human apoptotic neurons. Cell Death Differ 2006; 13: 285-292.

20. Schroder K, Tschopp J. The inflammasomes. Cell 2010; 140: 821-832.

21. Martinon F, Tschopp J. Inflammatory caspases and inflammasomes: master switches of inflammation. Cell Death Differ 2007; 14: 10-22.

22. Stutz A, Golenbock DT, Latz E. Inflammasomes: too big to miss. J Clin Invest 2009; 119: 3502-3511.

23. Shi F, Yang L, Kouadir M, Yang Y, Wang J, Zhou X et al. The NALP3 inflammasome is involved in neurotoxic prion peptide-induced microglial activation. J Neuroinflammation 2012; 9. 73

24. Lee HM, Kang J, Lee SJ, Jo EK. Microglial activation of the NLRP3 inflammasome by the priming signals derived from macrophages infected with mycobacteria. Glia 2013; 61: 441-452.

25. Frederick Lo C, Ning X, Gonzales C, Ozenberger BA. Induced expression of death domain genes NALP1 and NALP5 following neuronal injury. Biochem Biophys Res Commun 2008: 366: 664-669.

26. Liu F, Lo CF, Ning X, Kajkowski EM, Jin M, Chiriac C et al. Expression of NALP1 in cerebellar granule neurons stimulates apoptosis. Cell Signal 2004; 16: 1013-1021.

27. Abulafia DP, de Rivero Vaccari JP, Lozano JD, Lotocki G, Keane RW, Dietrich WD. Inhibition of the inflammasome complex reduces the inflammatory response after thromboembolic stroke in mice. J Cereb Blood Flow Metab 2009; 29: 534-544.

28. de Rivero Vaccari JP, Lotocki G, Alonso OF, Bramlett HM, Dietrich WD, Keane RW. Therapeutic neutralization of the NLRP1 inflammasome reduces the innate immune response and improves histopathology after traumatic brain injury. J Cereb Blood Flow Metab 2009; 29: 1251-1261.

29. de Rivero Vaccari JP, Lotocki G, Marcillo AE, Dietrich WD, Keane RW. A molecular platform in neurons regulates inflammation after spinal cord injury. J Neurosci 2008; 28: 3404-3414.

30. Fann DY, Lee SY, Manzanero S, Tang SC, Gelderblom M, Chunduri P et al. Intravenous immunoglobulin suppresses NLRP1 and NLRP3 inflammasome-mediated neuronal death in ischemic stroke. Cell Death Dis 2013; 4: e790.

31. Silverman WR, de Rivero Vaccari JP, Locovei S, Qiu F, Carlsson SK, Scemes E et al. The pannexin 1 channel activates the inflammasome in neurons and astrocytes. J Biol Chem 2009: 284: 18143-18151.

32. Li Q, Tian Y, Wang ZF, Liu SB, Mi WL, Ma HJ et al. Involvement of the spinal NALP1 inflammasome in neuropathic pain and aspirin-triggered-15-epi-lipoxin A4 induced analgesia. Neuroscience 2013; 254: 230-240.

33. Mawhinney LJ, de Rivero Vaccari JP, Dale GA, Keane RW, Bramlett HM. Heightened inflammasome activation is linked to age-related cognitive impairment in Fischer 344 rats. BMC Neurosci 2011; 12: 123.

34. Zou J, Crews FT. Inflammasome-IL-1beta signaling mediates ethanol inhibition of hippocampal neurogenesis. Front Neurosci 2012; 6: 77.

35. Adamczak SE, de Rivero Vaccari JP, Dale G, Brand lii FJ, Nonner D, Bullock M et al. Pyroptotic neuronal cell death mediated by the AIM2 inflammasome. J Cereb Blood Flow Metab 2014; 34: 621-629.

36. Kummer JA, Broekhuizen R, Everett H, Agostini L, Kuijk L, Martinon F et al. Inflammasome components NALP 1 and 3 show distinct but separate expression profiles in human tissues suggesting a site-specific role in the inflammatory response. J Histochem Cytochem 2007; 55: 443-452.

37. Ramaswamy V, Walsh JG, Sinclair DB, Johnson E, Tang-Wai R, Wheatley BM et al. Inflammasome induction in Rasmussen's encephalitis: cortical and associated white matter pathogenesis. J Neuroinflammation 2013; 10: 152.

38. Martinon F, Burns K, Tschopp J. The inflammasome: a molecular platform triggering activation of inflammatory caspases and processing of prolL-beta. Mol Cell 2002; 10: 417-426.

39. Qin L, Wu X, Block ML, Liu Y, Breese GR, Hong JS et al. Systemic LPS causes chronic neuroinflammation and progressive neurodegeneration. Glia 2007; 55: 453-462. 
40. LeBlanc A. Increased production of $4 \mathrm{kDa}$ amyloid beta peptide in serum deprived human primary neuron cultures: possible involvement of apoptosis. J Neurosci 1995; 15: 7837-7846.

41. LeBlanc A, Liu H, Goodyer C, Bergeron C, Hammond J. Caspase-6 role in apoptosis of human neurons, amyloidogenesis, and Alzheimer's disease. J Biol Chem 1999; 274: 23426-23436.

42. Moore BD, Chakrabarty P, Levites Y, Kukar TL, Baine AM, Moroni T et al. Overlapping profiles of Abeta peptides in the Alzheimer's disease and pathological aging brains. Alzheimers Res Ther 2012; 4: 18.

43. Walsh JG, Muruve DA, Power C. Inflammasomes in the CNS. Nat Rev Neurosci 2014; 15 84-97.

44. Halle A, Hornung V, Petzold GC, Stewart CR, Monks BG, Reinheckel T et al. The NALP3 inflammasome is involved in the innate immune response to amyloid-beta. Nat Immunol 2008; 9: 857-865.

45. Heneka MT, Kummer MP, Stutz A, Delekate A, Schwartz S, Vieira-Saecker A et al. NLRP3 is activated in Alzheimer's disease and contributes to pathology in APP/PS1 mice. Nature 2013; 493: 674-678.

46. Laudisi F, Spreafico R, Evrard M, Hughes TR, Mandriani B, Kandasamy M et al. Cutting edge: the NLRP3 inflammasome links complement-mediated inflammation and IL-1beta release. J Immunol 2013; 191: 1006-1010.

47. Pontillo A, Catamo E, Arosio B, Mari D, Crovella S. NALP1/NLRP1 genetic variants are associated with Alzheimer disease. Alzheimer Dis Assoc Disord 2012; 26: 277-281.
48. Tan MS, Tan L, Jiang T, Zhu XC, Wang HF, Jia CD et al. Amyloid-beta induces NLRP1-dependent neuronal pyroptosis in models of Alzheimer's disease. Cell Death Dis 2014; 5: e1382.

49. Kone-Paut I, Piram M. Targeting interleukin-1beta in CAPS (cryopyrin-associated periodic) syndromes: what did we learn? Autoimmun Rev 2012; 12: 77-80.

50. Bialer M, Johannessen SI, Levy RH, Perucca E, Tomson T, White HS. Progress report on new antiepileptic drugs: a summary of the Eleventh Eilat Conference (EILAT XI). Epilepsy Res 2013; 103: 2-30.

51. LeBlanc AC, Xue R, Gambetti P. Amyloid precursor protein metabolism in primary cell cultures of neurons, astrocytes, and microglia. J Neurochem 1996; 66: 2300-2310.

52. Lee AW, Champagne N, Wang X, Su XD, Goodyer C, Leblanc AC. Alternatively spliced caspase-6B isoform inhibits the activation of caspase-6A. J Biol Chem 2010; 285: 31974-31984.

53. Misiewicz M, Dery MA, Foveau B, Jodoin J, Ruths D, LeBlanc AC. Identification of a novel endoplasmic reticulum stress response element regulated by XBP1. J Biol Chem 2013; 288: 20378-20391.

54. Livak KJ, Schmittgen TD. Analysis of relative gene expression data using real-time quantitative PCR and the 2(-Delta Delta C(T)) Method. Methods 2001; 25: 402-408.

55. Hornung V, Ablasser A, Charrel-Dennis M, Bauernfeind F, Horvath G, Caffrey DR et al. AIM2 recognizes cytosolic dsDNA and forms a caspase-1-activating inflammasome with ASC. Nature 2009; 458: 514-518.

Supplementary Information accompanies this paper on Cell Death and Differentiation website (http://www.nature.com/cdd) 